colleagues, are not easily translated between different organ systems. Perhaps the growing sophistication of three-dimensional culture systems and organ-on-a-chip devices will, over time, aid the development of technologies for mechanical manipulation of adult organs. More advances in this exciting area of research will no doubt reveal fundamental aspects of adult organ maintenance and improve strategies for tissue engineering.
Jackson Liang and Lucy Erin O'Brien are in the Department of Molecular and Cellular Physiology, Stanford University School of Medicine, Stanford, California 94305, USA. e-mail:lucye@stanford.edu

1. Kumar, A., Placone, J. K. \& Engler, A. J. Development 144, 4261-4270 (2017).

2. Vining, K. H. \& Mooney, D. J. Nature Rev. Mol. Cell Biol. 18, 728-742 (2017).

3. He, L., Si, G., Huang, J., Samuel, A. D. T. \&
Perrimon, N. Nature 555, 103-106 (2018)

4. Wu, J., Lewis, A. H. \& Grandl, J. Trends Biochem. Sci. 42, 57-71 (2017)

5. Sallé, J. et al. EMBO J. 36, 1928-1945 (2017).

6. Deng, H., Gerencser, A. A. \& Jasper, H. Nature $\mathbf{5 2 8}$ 212-217 (2015).

7. Li, L. \& Clevers, H. Science 327, 542-545 (2010).

8. Yan, K. S. et al. Cell Stem Cell 21, 78-90 (2017).

9. Haber, A. L. et al. Nature 551, 333-339 (2017).

10. Barriga, F. M. et al. Cell Stem Cell 20, 801-816 (2017)

This article was published online on 7 February 2018.

\title{
Quantum
}

\section{upside-down cake}

\section{Exotic states of matter called topological superconductors have potential applications in quantum computing, but have been difficult to produce in more than one dimension. A way of overcoming this limitation has now been found.}

\section{CHIH-KANG SHIH \& ALLAN H. MACDONALD}

$\mathrm{O}$ ver the past decade, there has been explosive growth in the study of condensed-matter systems that have striking properties associated with unusual topologies ${ }^{1}$. The new kid on the block, experimentally speaking, is the topological superconductor. Like all superconductors, a topological one can transfer electric current without energy dissipation. But it also displays exotic excitations called Majorana modes, which are being investigated for use in quantum computing because they are extremely resistant to external interference ${ }^{2}$. Onedimensional topological superconductors have previously been reported $^{3,4}$. Writing in Nature Communications, Ménard et al. ${ }^{5}$ describe a technique for generating such superconductors in two dimensions.

Topological superconductors (TSCs) can be produced by combining three ingredients: ordinary superconductivity; strong coupling between the spins (magnetic moments) of electrons and their orbital motion; and the breaking of a fundamental symmetry of nature called time-reversal symmetry. The first-reported 1D TSC was realized using an ordinary superconductor, a nanoscale semiconducting wire that had strong spin-orbit coupling, and applied external magnetic fields ${ }^{3}$. Another 1D TSC was subsequently generated by placing ordered chains of magnetic atoms on the surface of superconducting lead, which has strong spin-orbit coupling ${ }^{4}$. In

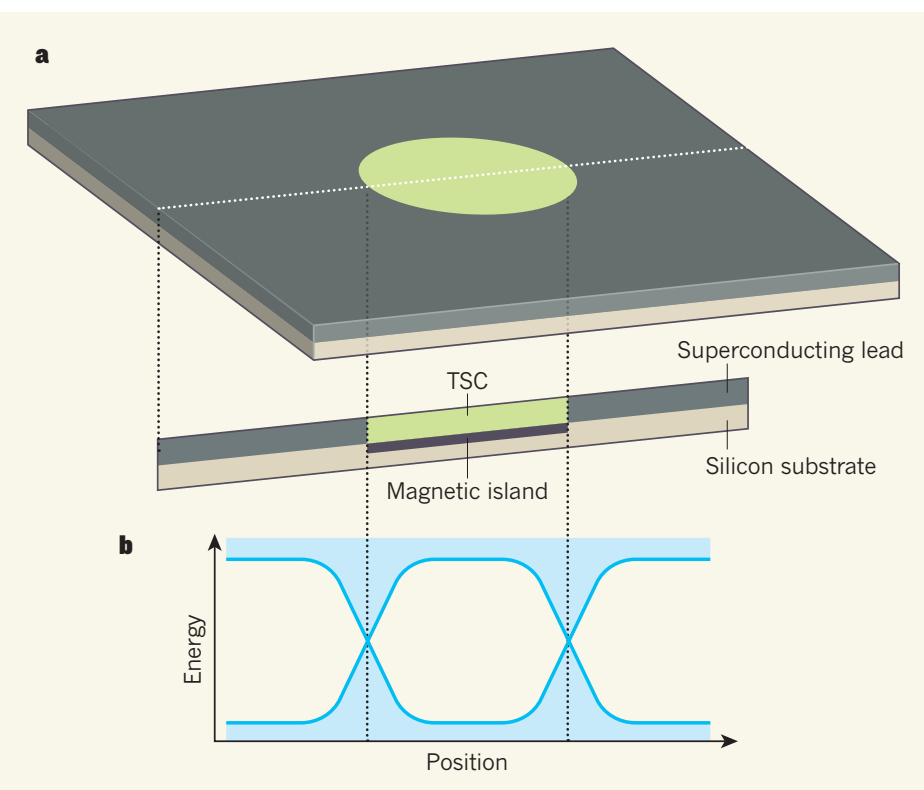

Figure 1 | Generation of a 2D topological superconductor. Ménard et al. ${ }^{5}$ report a technique for producing exotic states of matter called topological superconductors (TSCs) in two dimensions. Such materials exhibit superconductivity, whereby electric current can be transferred without energy dissipation, and show excitations called Majorana edge modes, which could be useful in quantum computing. a, Ménard and colleagues' experiment consists of a silicon substrate, structures called magnetic islands (for simplicity, a single island is shown here) and an atomically thin layer of superconducting lead. The authors report the generation of a TSC in the region of the lead directly above a magnetic island. b, Ménard et al. obtain an energy spectrum for their system that shows the possible energies that electrons can have (light blue) at different positions. They detect X-shaped features in the spectrum at positions corresponding to magneticisland boundaries that they interpret as evidence of Majorana edge modes. both cases, signatures of localized Majorana modes were observed at the ends of the TSC.

Unlike these 1D examples, a 2D TSC supports Majorana modes that are free to propagate ${ }^{1}$, a potentially desirable feature for quantum-computing applications. Such modes travel along the edge of the TSC and are chiral, meaning that they move in just one direction. This chiral property produces a characterismomentum space - a theoretical space that is mathematically equivalent to real space, which offers an alternative perspective of quantum systems by replacing positions with momenta. One method for producing a 2D TSC is to couple the excitations that exist on the surface of a 3D topological insulator to an ordinary $2 \mathrm{D}$ superconductor ${ }^{6,7}$. An alternative approach is to link a $2 \mathrm{D}$ superconductor that has strong spin-orbit coupling to a $2 \mathrm{D}$ magnetic system ${ }^{8}$.

Ménard and colleagues' work is probably the first successful realization of a 2D TSC based on the latter approach. The authors produced a $2 \mathrm{D}$ magneticsuperconductor composite system using a method known as epitaxy, whereby materials are grown on top of a substrate layer by layer. In this method, magnetic materials naturally separate into an array of isolated patches known as islands. The authors' experimental set-up consisted of a silicon substrate, an atomically thin film of superconducting lead, and magnetic islands made of cobalt.

Although ultrathin lead films are routinely grown on silicon substrates with atomic precision $^{9}$, the growth of magnetic islands on top of such films is technically challenging because the structural integrity of the film is often disrupted. Ménard et al. turned this strategy upside down: they grew their composite system in such a way that the magnetic islands formed underneath the superconducting-lead film (Fig. 1a). 
The authors knew that if a 2D TSC could be produced, it would be located in a region of the lead film directly above a magnetic island. The growth procedure would produce seamless lateral interfaces between the 2D TSC and the surrounding lead, and Majorana modes would be expected to propagate along these interfaces.

To look for evidence of such edge modes, the authors used a scanning tunnelling microscope to obtain an energy spectrum of electrons in the lead film across the putative interfaces. The microscope had a superconducting tip to maximize the spectral resolution. However, because scanning tunnelling microscopy is a tool operating in real space, it does not directly reveal the chiral nature of Majorana edge modes, which is evident only when the energy spectrum is measured in momentum space.

In the absence of exotic excitations, the realspace energy spectrum of a superconductor contains a gap - a range of energy values that electrons cannot have. Ménard et al. found that gaps in the material's energy spectrum were missing at positions corresponding to magnetic-island boundaries (Fig. 1b). The authors interpreted these features as evidence of exotic edge modes. They argue that these states are topological because they are resistant to the relatively strong disorder in the lead film. Furthermore, although non-topological states could give rise to a reduced gap, they would not necessarily remove the gap entirely. The observed X-shaped gap boundaries are therefore indicative of Majorana edge modes.

Ménard and colleagues' work provides strong, albeit indirect, evidence for 2D topological superconductivity in a $2 \mathrm{D}$ magneticsuperconductor composite system. The evidence is indirect because it cannot rule out the possibility that non-chiral edge modes are responsible for the observed X-shaped gap boundaries. If the chiral character of the edge modes is confirmed, the authors' system might be an excellent platform for studying Majorana states, which are of interest, not only for topological quantum computation, but also in elementary-particle physics ${ }^{10}$.

Chih-Kang Shih and Allan H. MacDonald are in the Department of Physics, University of Texas at Austin, Austin, Texas 78712, USA. e-mails: shih@physics.utexas.edu; macdpc@physics.utexas.edu

1. Hasan, M. Z. \& Kane, C. L. Rev. Mod. Phys. 82, 3045-3067 (2010)

2. Nayak, C., Simon, S. H., Stern, A., Freedman, M. \& Das Sarma, S. Rev. Mod. Phys. 80, 1083-1159 (2008).

3. Mourik, V. et al. Science 336, 1003-1007 (2012).

4. Nadj-Perge, S. et al. Science 346, 602-607 (2014)

5. Ménard, G. C. et al. Nature Commun. 8, 2040 (2017).

6. Fu, L. \& Kane, C. L. Phys. Rev. Lett. 100, 096407 (2008).

7. Xu, J.-P. Phys. Rev. Lett. 112, 217001 (2014)

8. Li, J. et al. Nature Commun. 7, 12297 (2016).

9. Nam, H. et al. Proc. Natl Acad. Sci. USA 113, 10513-10517 (2016)

10.Elliott, S. R. \& Franz, M. Rev. Mod. Phys. 87, 137-163 (2015).

This article was published online on 26 February 2018.

\section{BIOPHYSICS}

\section{Tight complexes from disordered proteins}

Charged groups on protein surfaces often take part in molecular interactions. Two unstructured proteins have been found to use charge complementarity to form a tight complex that has biologically useful kinetic properties. SEE ARTICLE P.61

\section{REBECCA B. BERLOW \& PETER E. WRIGHT}

1 The axiom 'opposites attract' applies to many aspects of life, including the many positively and negatively charged biological molecules that control the intricate cellular processes that enable an organism to survive. Even in the crowded environment of a cell, proteins can seek out their binding partners by using charged regions to attract oppositely charged molecules. This is certainly the case for the extreme example described by Borgia et al. ${ }^{1}$ on page 61 , in which a high degree of opposing charge in two binding partners that lack a defined 3D structure enables the partners to associate rapidly to form a tight complex, without the need for specific interactions between amino-acid residues. Remarkably, the complex forms without either binding partner adopting a defined structure, thereby revealing a previously unknown interaction mechanism for biological molecules.

The authors sought to characterize the binding between two highly charged proteins: negatively charged prothymosin- $\alpha$ (Pro-Ta) and the positively charged linker histone H1.0 (H1). Both Pro-Ta and H1 are intrinsically disordered, meaning that they do not adopt defined structures in solution, but remain flexible and accessible for binding interactions. Previous studies ${ }^{2}$ have shown that intrinsically disordered proteins typically lose some of their native flexibility when forming complexes, either by adopting a structure of their own, or by wrapping around a folded partner.

Surprisingly, Borgia et al. do not observe any gain of structure for either Pro-Ta or $\mathrm{H} 1$ on complex formation. Using a combination of nuclear magnetic resonance (NMR) spectroscopy, single-molecule fluorescence techniques and complementary computational approaches, the authors show that both proteins remain highly flexible in the complex.

Furthermore, Pro-T $\alpha$ and $\mathrm{H} 1$ associate with extremely high affinity at physiological salt concentrations (the dissociation constant for the complex is of the order of picomolar), even though their complex is highly disordered. Because the formation of complexes is driven by complementary charge interactions, the binding strongly depends on the salt concentration and becomes much weaker as the concentration is increased beyond the physiological range. Moreover, the authors find that amino-acid residues throughout Pro-Ta and $\mathrm{H} 1$ are affected similarly by binding. This implies that complex formation does not depend on the existence of specific binding sites in each of the proteins - instead, interactions are distributed widely over regions of opposite charge.

Charged amino-acid residues on the surface of globular proteins are commonly associated with binding 'hot spots' - localized
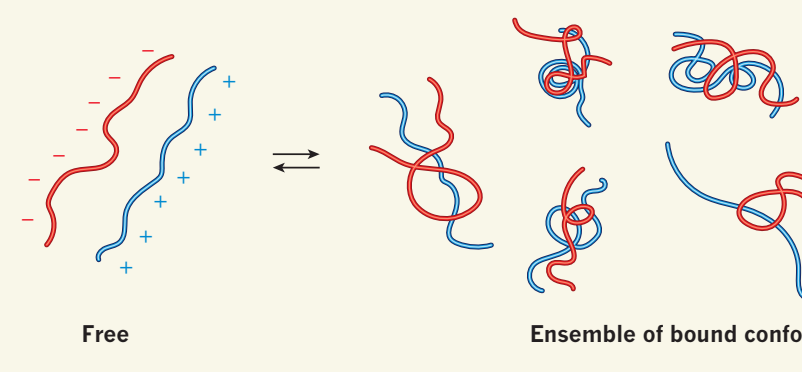

Ensemble of bound conformations

Figure $1 \mid$ Charge complementarity allows formation of tight complexes between disordered proteins. Borgia et al. ${ }^{1}$ studied the formation of a complex between two proteins that, in solution, lack a defined 3D structure: prothymosin- $\alpha$, which is negatively charged, and the linker histone H1.0, which is positively charged. The authors find that the complementarity of the charges on the two proteins enables them to bind reversibly and with extremely high affinity. This produces a large ensemble of bound protein conformations, many of which are adopted by only a few individual complexes and occur with approximately equal probability. 